\title{
ECOÉPIDÉMIOLOGIE de LA BORRÉLIOSE de LyME DANS LA RÉGION RHÔNE-ALPES Répartition, contexte écologique, relations avec la distribution d'Ixodes ricinus (Linné, 1758)
}

\author{
PICHOT J.*, GILOT B.**, SOULIER V.*, REY-COQUAIS A.*, DEGEILH B.*** et DOCHE B.****
}

Summary : ECOEPIDEMIOLOGICAL STUDY OF LYME BORRELIOSIS IN RHONE-ALPES AREA

The aim of the study was to precise the geographical repartition of Lyme borreliosis in the Rhône-Alpes district, to describe the ecological characteristics of the areas propitious to the disease, and to verify the vectorial competence of 1 . ricinus.

The cases of lyme disease were located by means of a questionnaire sent to 1156 physicians. The vectory role of I. ricinus was studied by two ways : firstly by searching a correlation between the geographical repartition of the tick and that of cases, secondly by proving the Borrelia infection of the tick.

Lyme disease is widely spread in the study area, mainly at the collinean level, its repartition is largely coinciding with that of 1 . ricinus which was found infected by B. burgdorferi (s.l.). However a few cases, located near the mediterranean area, set an unanswered problem.

KEY WORDS : Lyme borreliosis. Ixodes ricinus, Rhône-Alpes area.

\section{INTRODUCTION}

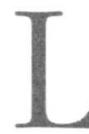

ocaliser les territoires où la maladie est susceptible de se manifester est une étape essentielle dans l'appréciation du risque épidémiologique. Dans le cas des maladies vectorielles, cette démarche n'a de véritable signification que si elle s'appuie sur l'étude des populations d'arthropodes vecteurs ("Précellence du vecteur", Rioux et al., 1979), dont l'existence est conditionnée par des exigences écologiques très précises. Ainsi, la répartition d'ensemble de la fièvre boutonneuse méditerranéenne en France apparaît-elle liée à la distribution de Rhipicephalus sanguineus (Latreille, 1806) (Gilot et al., 1990).

Les données sur la répartition de la borréliose de Lyme en France disponibles à ce jour (Doby, 1986; Dournon et al.,1986 et 1987; Pichot et al., 1990), tout imprécises qu'elles soient, permettent de penser que cette maladie est actuellement très répandue dans notre pays, comme elle l'est dans certains pays limi-

* Laboratoire de Parasitologie, Faculté de Pharmacie de Lyon.

** INSERM (U399) Laboratoire de Parasitologie, Faculté de Médecine de Marseille.

*** Laboratoire de Parasitologie, Faculté de Médecine de Rennes.

*****Laboratoire d'Écologie Végétale, Université Grenoble I.

\section{Résumé}

Ce travail a pour but d'étudier la répartition géographique de la maladie de Lyme dans la région Rhône-Alpes, de préciser les caractères écologiques des zones favorables à la maladie et de vérifier le rôle vecteur $d^{\prime}$ '. ricinus.

Les cas de maladie de Lyme ont été localisés grâce à un questionnaire adressé à 1156 médecins généralistes et spécialistes exerçant dans la zone d'étude. Le rôle vecteur d'l. ricinus a été étudié d'une part par la recherche d'une concordance entre la répartition géographique de cette espèce et celle des cas observés, d'autre part en recherchant B. burgdorferi (s.l.) dans les populations de tiques. La maladie de Lyme est largement répandue dans la zone d'étude, principalement à l'étage collinéen; sa répartition coïncide en grande partie avec celle $d^{\prime}$ l. ricinus dont l'infestation par B. burgdorferi a été démontrée. Cependant quelques cas, proches de la région méditerranéenne posent des problèmes non résolus.

MOTS CLES : borréliose de Lyme. |xodes ricinus, région Rhône-Alpes.

trophes (ainsi, Aeschlimann et ses collaborateurs l'ont bien montré, dans le cas de la Suisse, en 1986). Dans la mesure où la tique Ixodes ricinus (Linné, 1758), considérée en France comme son vecteur essentiel (Anderson et al., 1986), est loin d'y être uniformément répartie (Gilot et al., 1994), on peut prévoir une distribution non homogène de la borréliose.

La présente étude a pour but, d'abord, de préciser la répartition de la borréliose de Lyme dans la région Rhône-Alpes et les régions avoisinantes, soit 10 départements couvrant approximativement une surface de $30000 \mathrm{~km}^{2}$. Il s'agit d'un ensemble particulièrement diversifié du point de vue écologique. Nous avons essayé ensuite de caractériser, d'un point de vue écologique, les principaux territoires sur lesquels des microfoyers de maladie ont été mis ainsi en évidence et, enfin, de confirmer le caractère plausible du rôle vectoriel d'Ixodes ricinus, dans la genèse de ces microfoyers et la transmission de la borréliose à l'homme, en prouvant l'infestation de cette tique par Borrelia burgdorferi, sur le territoire d'étude et en vérifiant la présence du vecteur dans l'environnement proche des différents microfoyers détectés. 


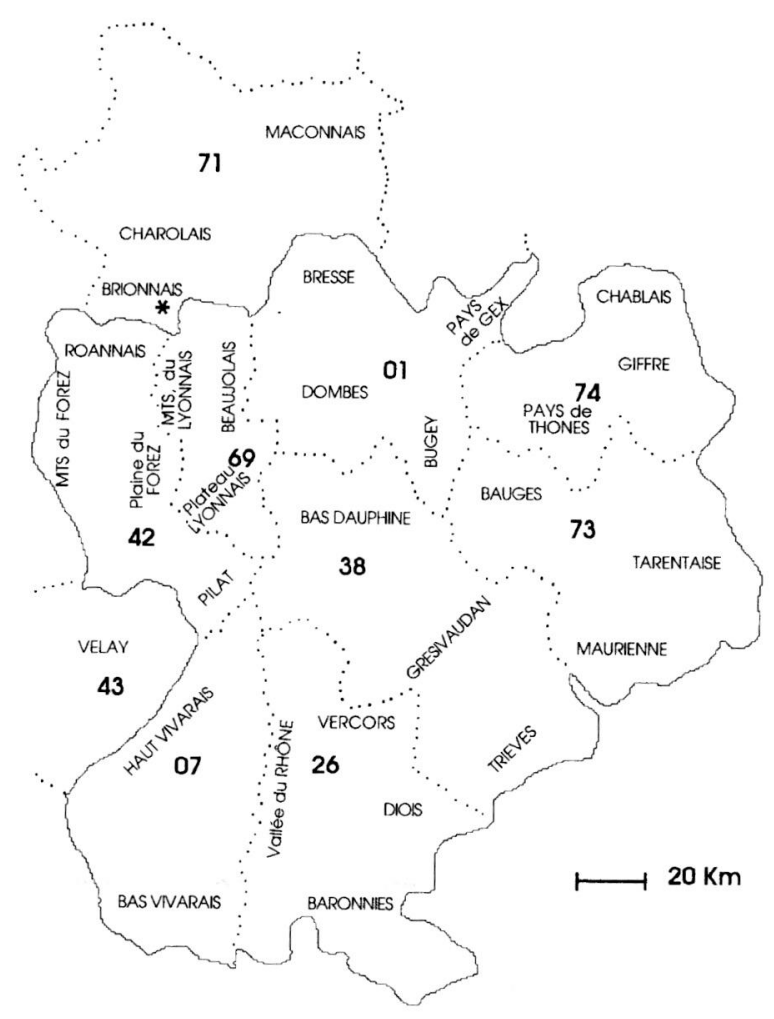

Fig. 1. - Zone d'étude Limites de la région Rhône-Alpes -..... Limites des départements * Chaufailles (71)

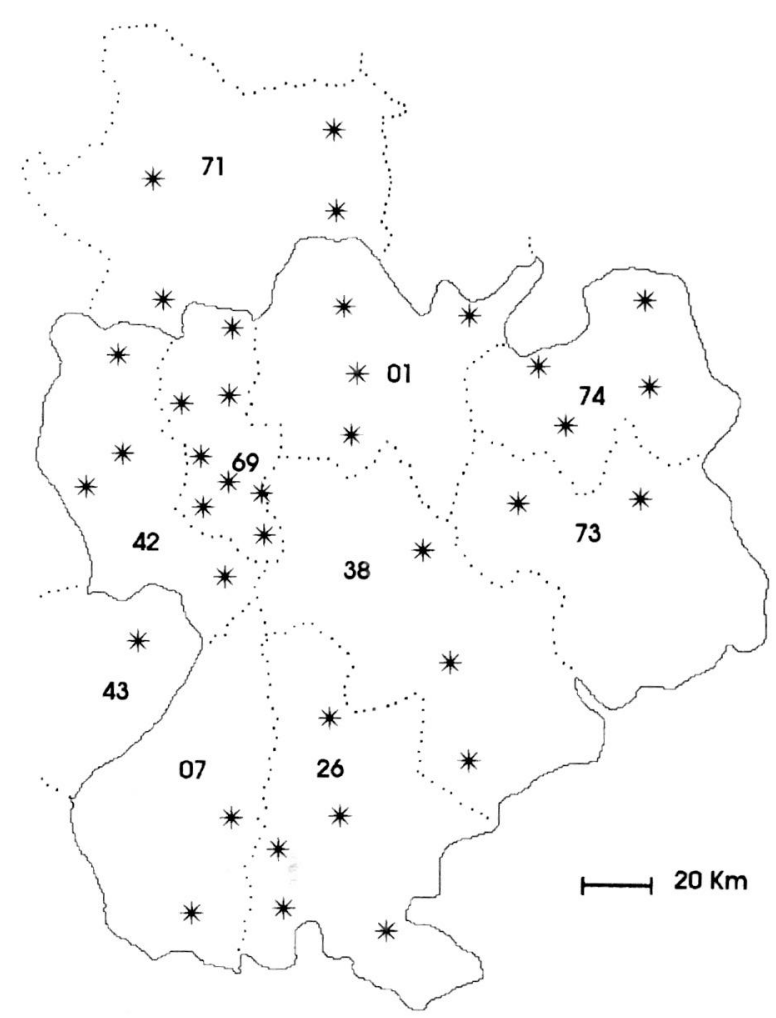

Fig. 2. - Répartition géographique des cas de maladie de Lyme

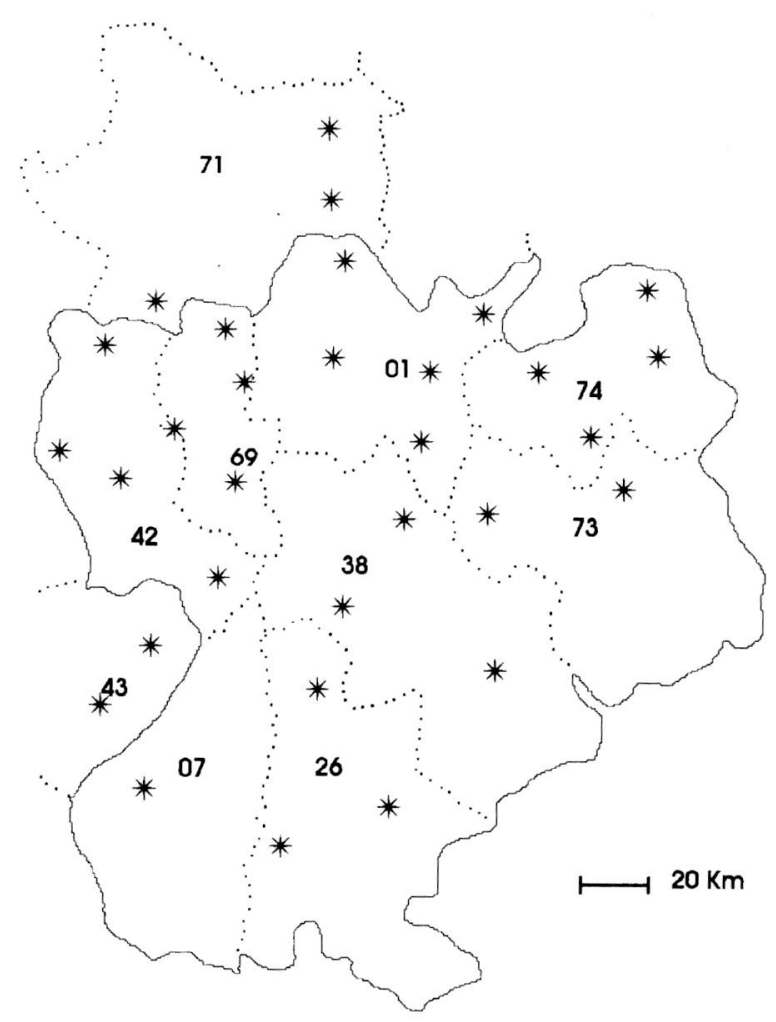

Fig. 3. - Répartition géographique de Ixodes ricinus 


\section{MÉTHODES}

\section{Territoire Étudié}

Il correspond aux départements suivants : HauteSavoie (74), Savoie (73), Isère (38), Ain (01), Saôneet-Loire (71), Rhône (69), Loire (42), Haute-Loire (43), Drôme (26), Ardèche (07) dans lesquels les régions naturelles ont été définies par Dupias et Rey (1985) (fig. 1).

\section{DonnéES NOSOlOGIQUeS}

Elles reposent sur les réponses à un questionnaire adressé à 1156 médecins susceptibles d'avoir porté le diagnostic de borréliose de Lyme : généralistes ou spécialistes (dermatologues, neurologues, rhumatologues, cardiologues) exerçant, soit en cabinet privé, soit en milieu hospitalier, ainsi qu'aux biologistes pratiquant la sérologie de la maladie dans le territoire exploré.

Les critères qui nous ont permis de sélectionner, parmi tous les cas proposés, ceux que l'on pouvait retenir comme d'authentiques cas de borréliose sont les suivants :

- érythème chronique migrant avec notion de piqûre de tique, que la sérologie soit positive ou négative;

- manifestations cliniques, isolées ou associées (rhumatologiques, neurologiques, cardiaques...) évoquant la phase secondaire de la maladie, accompagnées d'une sérologie positive, avec ou sans notion de piqûre de tique. Les sérologies ont été effectuées en immunofluorescence indirecte en utilisant comme antigène la souche américaine B31. Les seuils de positivité sont les suivants : immunofluorescence indirecte : IgG : $1 / 128$ ou $1 / 160$, selon les laboratoires; IgM : 1/32; Test Elisa : 1/256 ou 320 selon la dilution choisie. Le Western-blot a été pratiqué dans les cas les plus récents pour mettre en évidence les anticorps anti-protéine P39, spécifique de Borrelia burgdorferi.

\section{DONnÉES ACAROLOGIQUES}

C'est la région française qui a bénéficié des études les plus récentes et les plus extensives : la répartition d'Ixodes ricinus telle qu'elle est évoquée ici repose sur un dense réseau d'informations dont l'essentiel a été fourni par les travaux de Roman et al. (1973) pour "la région lyonnaise", ceux de Gilot (1985) pour l'ensemble des Alpes françaises et leur avant-pays et de Gilot et al. (1989), pour le rebord oriental du Massif Central. Une enquête globale, à l'échelle de la France, effectuée récemment (Gilot et al., 1994) a apporté des informations complémentaires.

Un supplément d'informations a été apporté récem- ment par les prospections effectuées par Crom (1990) pour le Velay, Conan (1991) pour la Saône-et-Loire, Nevers (1987) pour le Roannais et le Brionnais, Vuillermet (1991) pour la Savoie et la Haute-Savoie, Farjaud (1991) pour la Haute-Loire, Halleguen (1991) pour la Drôme et l'Ardèche.

\section{DONnÉES BACTÉrIOLOGIQUES}

Nous n'avons pas cherché à prouver la présence du germe dans toutes les populations d'Ixodes ricinus détectées mais dans six d'entre elles récoltées dans des sites où des cas de borréliose de Lyme avaient été localisés avec certitude : 103 tiques ont été récoltées à Chauffailles (71), 26 dans la Forêt de la Réna, près de Bourg-en-Bresse (01), dans les Monts du Lyonnais : 36 à Avèze, 21 au Col des Cassettes, 24 à Cublize (69) et 20 à Anthy (74), soit au total 230 tiques (Rey-Coquais, 1991).

L'isolement de Borrelia burgdorferi a été pratiqué selon une technique classique, sur le milieu de Kelly modifié (milieu BSKII) : 97 tubes ont été ensemencés, chacun avec deux femelles, deux mâles ou trois nymphes.

L'identification de la borrélie a été réalisée grâce à trois techniques complémentaires, en comparaison avec deux souches de référence : souche B31 (américaine, isolée d'Ixodes dammini), souche ATC 35211 (européenne, isolée d'Ixodes ricinus):

- immunofluorescence à l'aide d'anticorps monoclonaux : H 5332 et H3TS anti-surface protein A (Osp A) de $31 \mathrm{Kd}$ et H 9724 anti-flagelline, protéine de $41 \mathrm{kd}$ commune à toutes les Borrelia (ces anticorps ont été fournis par A. Barbour, University of Texas, Health Science Center, San Antonio);

- électrophorèse en gel de polyacrylamide (PAGESDS), à pH 8,8 avec coloration des protéines par le bleu de Coomassie.

- Western-blot, après séparation par électrophorèse et transfert sur une membrane de nitrocellulose, les protéines sont révélées à l'aide des anticorps déjà cités pour l'immunofluorescence (Pichot et al., à paraître).

\section{RESULTATS}

\section{RÉPARTITION GÉOGRAPHIQUE DES CAS DE BORRÉ-}

\section{LIOSE DE LYME SUR LE TERRITOIRE D'ÉTUDE}

Cent trente-quatre cas ont été retenus. On observe la prédominance des formes dermatologiques, phase primaire de la maladie ( $68 \%$ des cas). Près des troisquarts des cas (67\%) ont pu être localisés avec une quasi-certitude, à l'intérieur d'un cercle de $15 \mathrm{~km}$ de 
rayon, précision habituellement suffisante, à l'échelle de l'étude, pour donner une définition écologique valable du territoire de contamination. Aucun des départements inventoriés n'est indemne (fig. 2).

Les principales zones et régions naturelles intéressées sont les suivantes : Charolais, Brionnais, Monts du Lyonnais, Haut-Beaujolais, Velay, Dombes, Bugey, Terres Froides, Plaine molassique péri-alpine (prolongement de la plaine suisse, elle aussi très contaminée, Aeschlimann et al., 1986), Trièves... Les sites de contamination présomptifs s'étagent de 180 à 1200 mètres (tableau I). Un maximum de cas (près de 95 \%) se situe dans le collinéen (de 180 à 800 mètres), et seulement $5 \%$ à l'étage montagnard. A l'étage collinéen, la répartition n'est pas homogène : la quasitotalité des zones de contamination (98\%) est localisée au-dessous de 500 mètres.

\section{ENVIRONNEMENT BOTANIQUE ET CARACTÉRISATION ÉCOLOGIQUE DES ZONES OU LA MALADIE SE MANI- FESTE}

Les unités phytosociologiques concernées se rapportent essentiellement aux chênaies (chênaies à charme et chênaies acidophiles) et plus accessoirement aux hêtraies ou à des groupements de substitution, telle la pinède à pin sylvestre. Le tableau I résume les composantes écologiques des principales entités géographiques favorables à la borréliose.

Comparaison ENTRE la Distribution DES CAS DE BORRELIOSE DE LYME ET CELLE DES POPULATIONS D'IXODES RICINUS

Dans l'ensemble, la répartition des cas de maladie de Lyme s'inscrit dans l'aire de répartition d'Ixodes ricinus, telle qu'on peut l'évoquer d'après les différents travaux évoqués plus haut (fig. 3). Cependant, une approche écologique comparative doit considérer la répartition du vecteur et celle de la maladie dans chacun des trois étages suivants : collinéen, montagnard, supra méditerranéen.

À l'étage collinéen :

La concordance est excellente. Rappelons que, dans le secteur d'étude, les populations d'Ixodes ricinus connaissent à cet étage une large diffusion sur le plan chorologique mais également phytosociologique, la plupart des groupements végétaux étant colonisés. De fait, il n'existe pas de microfoyer de maladie qui s'inscrive dans une zone où la tique n'a pu être mise en évidence.

\section{À l'étage montagnard :}

La concordance se vérifie encore en grande partie. Rappelons que seule la partie basse de cet étage, jusque vers 1200 mètres, peut être colonisée par l'espèce. Dans notre enquête, aucun cas de borréliose de Lyme n'a été contracté plus haut; cependant, cette concordance vecteur-maladie ne se vérifie, à cet étage, que sur la bordure orientale du Massif Central : les cas de maladie s'y étagent de 200 à 1200 mètres, tout comme les sites de distribution connus d'Ixodes ricinus. La limite haute se situe en Haute-Loire, dans le Velay. Par contre, dans les Alpes, d'après les données en notre possession, aucun site de contamination ne se situe avec certitude au-dessus de 1000 mètres. Cependant, la tique a souvent été détectée à cette altitude, surtout dans les Préalpes (Vercors, Chartreuse, Bornes,...) (Gilot, 1985).

\section{À l'étage supra-méditerranéen :}

Dans la partie la plus méridionale de la zone d'étude (partie Sud des départements de la Drôme et de l'Ardèche), la situation est tout autre. La présente enquête a permis de mettre l'accent sur quelques cas, dont le biotope de contamination paraissait se situer soit dans l'étage supra-méditerranéen, soit même dans l'étage méditerranéen (cas peu fréquents). Or, on connaissait la très grande rareté et les effectifs particulièrement modestes des populations d'Ixodes ricinus de la vallée du Rhône et ses abords (Gilot, 1985). De ce fait, des enquêtes locales, sur les lieux présumés de contamination par Borrelia burgdorferi, ont été programmées, pour tenter de détecter des populations d'Ixodes ricinus, si modiques soient-elles. Elles ont permis de mettre en évidence trois types de situation :

a) Cas apparemment contracté dans le supra-méditerranéen (région de Montélimar) et détection d'une population modique d'Ixodes ricinus sur un territoire proche (aire de repos de Savasse, sur autoroute A7) dans une enclave frâ̂che à peupliers blancs (Populetum albae) (Halleguen, 1991).

b) Cas apparemment contracté dans le supra-méditerranéen (ou le méditerranéen) (région de Joyeuse) et découverte de l'espèce vectrice, à moins de 30 kilomètres, sur les contreforts du Massif Central (Malons, 750 mètres) : ce biotope de l'étage submontagnard est, selon la nomenclature adoptée par Couteaux (1974), inséré dans la série du châtaignier-chêne sessile, qui fait la transition entre le domaine supraméditerranéen et la hêtraie subatlantique. Du fait de l'impossibilité de réinterroger la famille de l'enfant victime de la borréliose (famille belge en vacances dans l'Ardèche) nous n'avons pas pu vérifier que le site de contamination présomptif correspondait à la rẻalité.

c) Cas contracté, selon tout vraisemblance, dans l'étage méditerranéen, mais impossibilité de mettre en évidence l'espèce vectrice dans un périmètre proche. 


\begin{tabular}{|c|c|c|c|c|c|c|}
\hline $\begin{array}{l}\text { Entités } \\
\text { géographiques }\end{array}$ & Etage(s) & Série(s) de végétation & Altitude & $\begin{array}{l}\text { Pourcentage } \\
\text { de Forêts }\end{array}$ & $\begin{array}{l}\text { Températuremensudle } \\
\text { moyenne }\end{array}$ & $\begin{array}{l}\text { Précipitations } \\
\text { annuelles }\end{array}$ \\
\hline Charolais & Collinéen & $\begin{array}{l}\text { Série acidophile du Chêne sessile } \\
\text { Série neutrophile du Chêne sessile }\end{array}$ & 250 à $500 \mathrm{~m}$ & 10 à $20 \%$ & $10^{\circ} \mathrm{C}$ & 800 à 1000 mm \\
\hline Brionnais & Collinéen & $\begin{array}{l}\text { Série du Chêne sessile } \\
\text { neutrophile - acidophile }\end{array}$ & 250 à $440 \mathrm{~m}$ & $5 \%$ & $10^{\circ} \mathrm{C}$ & 700 à $900 \mathrm{~mm}$ \\
\hline Monts du Beaujolais & Collinéen & $\begin{array}{l}\text { Série acidophile des Chênes } \\
\text { (Chêne sessile) }\end{array}$ & 200 à $1000 \mathrm{~m}$ & $30 \%$ & 9 à $10^{\circ} \mathrm{C}$ & $\begin{array}{l}900 \text { à } 1100 \mathrm{~mm} \\
1100 \text { à } 1500 \mathrm{~mm}\end{array}$ \\
\hline Bresse & Collinéen & Série du Chêne pédonculé & 100 à $250 \mathrm{~m}$ & 10 à $15 \%$ & $11^{\circ} \mathrm{C}$ & $800 \mathrm{~mm}$ à $1100 \mathrm{~mm}$ \\
\hline Dombes & Collinéen & Chêne pédonculé & 240 à $500 \mathrm{~m}$ & 10 à $15 \%$ & $10^{\circ} \mathrm{C}$ & 1000 à 1100 mm \\
\hline Bas Bugey & Collinéen & $\begin{array}{l}\text { - Chênaie à Charme } \\
\text { - Série delphino-jurassienne } \\
\text { du Chêne pubescent }\end{array}$ & 400 à 1000 m & $\begin{array}{l}20 \% \\
40 \text { à } 50 \% \text { en } \\
\text { montagne }\end{array}$ & $8^{\circ}$ à $10^{\circ} \mathrm{C}$ & $\begin{array}{c}900 \mathrm{~mm} \\
1500 \mathrm{~mm} \text { en altitude }\end{array}$ \\
\hline Monts du Lyonnais & Collinéen & $\begin{array}{l}\text { Série de la Chênaie acidophile } \\
\text { Série du Hêtre en altitude }\end{array}$ & 350 à $900 \mathrm{~m}$ & $\begin{array}{l}10 \% \text { très } \\
\text { boisé en haut }\end{array}$ & $9^{\circ}$ à $10^{\circ} \mathrm{C}$ & 800 à $1200 \mathrm{~mm}$ \\
\hline Plateau Lyonnais & $\begin{array}{l}\text { Collinéen } \\
\text { Collinéo- } \\
\text { montagnard }\end{array}$ & $\begin{array}{l}\text { Série acidophile des Chênes } \\
\text { Série du Hêtre }\end{array}$ & $\begin{array}{l}240 \mathrm{~m} \\
500 \mathrm{~m}\end{array}$ & $10 \%$ & $10^{\circ}$ à $11^{\circ} \mathrm{C}$ & 800 à 1000 mm \\
\hline Velay & $\begin{array}{l}\text { Collinéo- } \\
\text { montagnard }\end{array}$ & $\begin{array}{l}\text { Végétation de moyenne montagne } \\
\text { Pinède à Pin sylvestre } \\
\text { Chênaie acidophile }\end{array}$ & 400 à 1000 m & $20 \%$ & $8^{\circ}$ à $9^{\circ} \mathrm{C}$ & 600 à $1000 \mathrm{~mm}$ \\
\hline Bas Dauphiné & Collinéen & $\begin{array}{l}\text { Chênaie acidophile } \\
\text { Chênaie à Charme }\end{array}$ & 250 à $750 \mathrm{~m}$ & 10 à $20 \%$ & $10^{\circ} \mathrm{C}$ & 900 à $1000 \mathrm{~mm}$ \\
\hline $\begin{array}{l}\text { Plaine molassique } \\
\text { périalpine }\end{array}$ & $\begin{array}{l}\text { Collinéo- } \\
\text { montagnard }\end{array}$ & $\begin{array}{l}\text { Série du Charme } \\
\text { Série du Hêtre } \\
\text { Série acidophile du Chêne sessile }\end{array}$ & $\begin{array}{l}400 \text { à } 500 \mathrm{~m} \\
1100 \mathrm{~m}\end{array}$ & $\begin{array}{c}5 \% \\
30 \text { à } 40 \%\end{array}$ & $\begin{array}{l}10^{\circ} \mathrm{C} \text { bord du lac } \\
8^{\circ} \mathrm{C} \text { en altitude }\end{array}$ & $\begin{array}{l}800 \mathrm{~mm} \\
1600 \mathrm{~mm}\end{array}$ \\
\hline Trièves & $\begin{array}{l}\text { Collinéo- } \\
\text { montagnard }\end{array}$ & $\begin{array}{l}\text { Série du Chêne pubescent } \\
\text { Série du Hêtre } \\
\text { Pinède à Pin sylvestre }\end{array}$ & $\begin{array}{l}500 \text { à } 1000 \\
\text { (au dessus : } \\
\text { montagne) }\end{array}$ & $20 \%$ & $8^{\circ}$ à $9^{\circ} \mathrm{C}$ & $\begin{array}{c}800 \text { à } 1200 \mathrm{~mm} \\
1500 \mathrm{~mm} \text { en altitude }\end{array}$ \\
\hline
\end{tabular}

Tableau I - Principales entités géographiques et phytoécologiques concernées par la borréliose de Lyme. 


\section{RÉSUltat DE LA RECHERCHE BACTÉRIOLOGIQUe}

Seules les tiques provenant de Chauffailles (71) ont permis l'isolement de spirochètes identifiés comme étant Borrelia burgdorferi (s.1.) : sur 44 tubes ensemencés, 21 ont donné une culture positive.

\section{DISCUSSION}

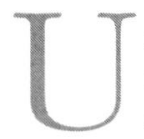
ne telle étude ne saurait donner une idée même approchée - de la fréquence de la maladie sur le territoire d'étude, et, moins encore, permettre une comparaison du niveau d'endémie de la maladie dans les diverses régions naturelles dont il est constitué. Elle permet par contre d'avoir une première idée des territoires occupés par la maladie et de préciser son amplitude écologique et paysagère.

Cette enquête, qui a bénéficié d'une forte participation des médecins interrogés (54\% de réponses) met en évidence la grande extension de la borréliose de Lyme dans la région Rhône-Alpes, aussi diversifiée soit-elle sur le plan écologique. La dispersion des cas dûment diagnostiqués est d'autant plus remarquable qu'il s'agit d'une maladie "nouvelle" dont le diagnostic est loin d'être aisé et qui peut encore passer inaperçue.

Doby (1986) a souligné, grâce à une enquête homologue, auprès des seuls dermatologues installés dans 18 départements du Nord-Ouest de la France, la très large extension de la borréliose sur cette partie de notre territoire. Dans ce cas, l'homogénéité climatique, la grande similitude des formations végétales rencontrées (Doche et al., 1993), expliquent la large diffusion de la tique vectrice, Ixodes ricinus, démontrée récemment par Degeilh et al. (sous presse).

Sur le territoire abordé dans le cadre de la présente étude, foncièrement différent, et beaucoup plus contrasté du point de vue écologique, Ixodes ricinus, dont nous avons prouvé ici l'infection par Borrelia burgdorferi, représente également, selon toute vraisemblance, le vecteur principal de la maladie. Il existe, en effet, une concordance chorologique troublante entre la répartition des "microfoyers" décelés et celle de ce vecteur, tant sur le plan horizontal que vertical. Le contexte écologique est cependant tout autre, la distribution de la tique étant, dans la région Rhône-Alpes, beaucoup moins homogène que dans le Nord-Ouest de la France. Il est notable que cette distribution "en mosaïque" du vecteur, soulignée par Gilot (1985), ne nuise pas à la diffusion de la maladie, largement distribuée, en dépit du fait que toutes les formations végétales sont loin de lui être favorables et que les groupements végétaux propices sont d'étendue très inégale.
Les résultats de cette enquête paraissent montrer que la borréliose existe pratiquement partout où des populations d'Ixodes ricinus ont été détectées, que ces populations soient abondantes, comme c'est le cas dans la Dombes ou le Bugey (Gilot et al., 1979), ou beaucoup plus modiques, région grenobloise, Trièves, (Gilot, 1985), vallée du Rhône au niveau de Montélimar (Halleguen, 1991). Ce fait traduit vraisemblablement d'une part la forte affinité d'Ixodes ricinus pour l'homme, soulignée par Gilot et Marjolet (1982), et d'autre part la fréquence d'infestation des populations du vecteur par la Borrelia, qu'il s'agira de prouver dans une étude ultérieure. On sait que c'est le cas dans un pays voisin, la Suisse (Aeschlimann et al., 1986).

Les résultats de cette étude montrent que certains groupements végétaux abritent régulièrement des sites de contamination; telles sont à l'étage collinéen, les chênaies à charme et les chênaies acidophiles. De tels groupements peuvent être considérés comme des "indicateurs épidémiologiques". Pour d'autres groupements appartenant à l'étage montagnard (hêtraies...) ou à l'étage supra-méditerranéen (chênaie pubescente) les résultats sont à parfaire avant qu'on puisse les considérer comme extrapolables.

En ce qui concerne les hêtraies, il parait exister une contradiction entre les résultats négatifs obtenus dans les Alpes et ceux positifs constatés sur le rebord oriental du Massif Central. Ainsi cette enquête n'a pas permis d'apporter la preuve de l'existence de cas de borréliose contractés à plus de 1000 mètres d'altitude dans les Alpes. Cette donnée doit être interprétée avec prudence car les modalités de l'enquête n'ont pas permis de répertorier tous les cas existant. Ces premiers résultats pourraient tout au plus signifier une plus grande rareté des cas quand s'accroît l'altitude ; ce qui pourrait s'expliquer soit par une diminution des populations d'Ixodes ricinus, constatée par différents auteurs en Europe Occidentale (Gilot 1979, Kaltenrieder 1985, Gourdon 1991) soit par une diminution de l'infection des tiques par le germe lorsqu'on s'élève en altitude, démontrée par Aeschlimann en Suisse en 1986.

On devra être encore plus prudent avant d'attribuer un caractère indicateur à l'étage supra-méditérranéen, série du chêne pubescent ou séries homologues de cet étage (Ozenda, 1979) présentes dans tous les pays du bassin méditérranéen occidental.

L'intervention majeure d'Ixodes ricinus, dans la genèse des foyers de la région considérée, n'interdit d'ailleurs pas de penser que d'autres espèces soient susceptibles d'intervenir à ses côtés, comme vecteurs mineurs : tel peut-être le cas de Pholeoixodes hexagonus (Leach, 1815) ou même de Dermacentor reticu- 
latus (Fabricius, 1794) (Kahl et al., 1992; Tremel, 1993), espèces dont on sait qu'elles peuvent se fixer sur l'homme (Gilot et Marjolet, op. cit.). Ces espèces sont susceptibles de se développer sur les mêmes territoires qu'Ixodes ricinus ou des territoires proches (Gilot, 1985). Rappelons que c'est à la première de ces espèces qu'a été attribuée la responsabilité du cas historique de Garin et Bujadoux (1922) et qu'aucun argument n'incite à penser à une erreur de détermination. Récemment, l'infestation naturelle de Pholeoixodes hexagonus a été démontrée (Liebisch et al., 1989; Doby et al., 1991) et ses possibilités de transmission prouvées expérimentalement (Gern et al., 1991).

\section{REMERCIEMENTS}

$\mathrm{N}$ ous remercions bien vivement le Professeur Doby qui nous a suggéré cette étude et qui a bien voulu confier à l'un de nous son modèle d'enquête auprès des médecins.

Cette étude a été réalisée, dans le cadre d'un contrat INSERM (Contrat de Recherche Externe No 900.801) et a, par surcroît, bénéficié d'une subvention de la Région Rhône-Alpes.

\section{RÉFÉRENCES}

Aeschlimann A., Chamot E., Gigon F., Jeanneret J., Kesseler D. and Walther C. B. burgdorferi in Switzerland. Zbl. Bakt. Hyg., 1986, A 263, 450-458.

Anderson J.F., Doby J.M., Couatarmanach A., Hyde F.W. et JoHnson R.C. Différences antigéniques entre des souches de Borrelia burgdorferi isolées d'Ixodes ricinus en Bretagne. Médecine et Maladies Infectieuses, 1986, 16, 171-175.

CONAn C. Les tiques et la maladie de Lyme dans le Sud Bourguignon. Thèse de Doctorat en Pharmacie, Lyon 1991.

Couteaux M. Essai de cartographie écologique du Bas Vivarais. Feuilles de Bessèges et Bourg-Saint-Andéol. Doc. Cart. Ecologique, 1974, XIII, 49-68

Crom M.P. Les tiques et la maladie de Lyme dans le Velay. Thèse de Doctorat en Pharmacie, Lyon 1990.

Degeilh B., Guiguen C., Gilot B., Doche B., Pichot J. et Beaucournu J.C. Répartition d'Ixodes ricinus (Linné, 1758) (Acarina : Ixodoidea) dans les groupements forestiers du Massif Armoricain. Acarologia, 1994, sous presse.

Doвy J.M. Résultats d'une enquête sur l'érythème migrant de Lipschütz dans l'ouest de la France. À propos de près de 700 cas diagnostiqués dans 18 départements pendant la dernière décade. Ouest Médical, 1986, 39, 167-172.

Doby J.M., Bigaignon G., Aubert M. el Imbert G. Ectoparasites du renard et borréliose de Lyme.
Recherches de Borrelia burgdorferi dans les tiques et les puces (Siphonaptères). Bulletin de la Société Française de Parasitologie, 1991, 9, 279-280.

Doche B., Gilot B., Degeilh B., Pichot J. et Guiguen C. Utilisation de l'indicateur végétal pour la cartographie d'une tique exophile à l'échelle d'un pays : l'exemple d'Ixodes ricinus en France. Annales de Parasitologie Humaine et Comparée, 1993, 68, 188-195.

Dournon E., Assous M., Candalot B. et Christol P. Aspects cliniques sérologiques et épidémiologiques de la maladie de Lyme en France. À propos de 154 cas. Bulletin épidémiologique Hebdomadaire, 1986, 9, 33-36.

Dournon E., Assous M. et Fourcade C. La maladie de Lyme en France (à propos de 272 cas). Bulletin épidémiologique hebdomadaire, 1987, 15, 57-58.

Dupias G. et Rey P. Document pour un zonage des régions phytoécologiques. Centre d'Ecologie des Ressources Renouvelables. CNRS, Toulouse, 1985, 40 p.

FARIAUD C. Les tiques et la maladie de Lyme dans la Haute Loire occidentale. Thèse de Doctorat en Pharmacie, Lyon 1991.

Garin Ch. et Bujadoux M. Paralysie par les tiques. Journal de Médecine de Lyon, 1922, 71, 765-767.

Gern L., Toutounei L.N., Hu C.M.et Aeschlimann A. Ixodes (Pholeoixodes) bexagonus an efficient vector of Borrelia burgdorferi in the laboratory. Medical and Veterinary Entomology, 1991, 5, 431-435.

Gilot B. Bases biologiques, écologiques et cartographiques pour l'étude des maladies transmises par les tiques (Ixodidae et Argasidae) dans les Alpes françaises et leur avant-pays. Thèse Doctorat-ès-sciences, Université Scientifique et Médicale de Grenoble, 1985, 535 p.

Gilot B., Guiguen C., Degeilh B., Doche B., Pichot J. and Beaucournu J.C. Phytoecological mapping of Ixodes ricinus as an approach to the distribution of Lyme Borreliosis in France. In "Lyme Borreliosis", J.S. Axford, DHE Rees ed, Plenum Press New York, 1994, 105-112.

Gilot B., Laforge M.L., Pichot J. and Raoult D. Relationships between the Rhipicephalus sanguineus complex ecology and mediterranean spotted fever epidemiology in France. European Journal of Epidemiology, $1990,6,357-362$

Gilot B., Marjolet M. Contribution à l'étude du parasitisme humain par les tiques (Ixodidae et Argasidae), plus particulièrement dans le sud-est de la France. Médecine et Maladies Infectieuses, 1982, 12, 340-351.

Gilot B., Pautou G., Moncada E., Lachet B. el Christin J.G. La cartographie des populations de tiques exophiles par le biais de la végétation : bases écologiques, intérêt épidémiologique. Doc. Cart. Ecologique, Grenoble, 1979, XXII, 65-80.

Gilot B., Pichot J. et Doche B. Tiques du Massif Central. I. Les populations de tiques parasites de carnivores et d'ongulés domestiques sur le rebord oriental du massif. Acarologia, 1989, 30, 8-24.

Gourdon V. Étude des tiques exophiles dans le département du Puy-de-Dôme. Thèse de Doctorat en Pharmacie, Clermont-Ferrand 1991. 
Halleguen C. Enquête sur la maladie de Lyme dans les départements de la Drôme et de l'Ardèche. Thèse de Doctorat en Pharmacie, Lyon 1991.

Kahl O., Janetzi C., Gray J.S., Stein J. and Bauch R.J. Tick infection rates with Borrelia: Ixodes ricinus versus Haemaphysalis concinna and Dermacentor reticulatus in two locations in Eastern Germany. Medical and Veterinary Entomology, 1992, 6, 363-366.

Kaltenrieder M., Hess E. and Aeschlimann A. Zum Vorkommen der Zecke Ixodes ricinus Linné (Ixodoïdea, Ixodidae) unter Schweiz. Revue Suisse de Zoologie, 1985 92, 685-694.

Liebish A., Olbrich S., Brand A., Liebish G. Mourettau and KIENITZ M. Natural infection of Ixodes hexagonus with Borrelia burgdorferi. Tierarztliche Umschau, 1989, 44, 809-810.

Nevers F. Tiques et maladie de Lyme. Enquête dans la plaine du Roannais et le Brionnais. Thèse de Doctorat en Pharmacie, Lyon 1987.

Ozenda P. Carte de la végétation des états membres du Conseil de l'Europe. Comité Européen pour la Sauvegarde de la Nature et des Ressources Naturelles, Strasbourg, 1979, $97 \mathrm{p}$.

Pichot J., Charhon A. Nevers F., Bancillon B. et Gilot B. Etude clinique, acarologique, bactériologique d'un cas de maladie de Lyme après contamination péridomestique. (à paraître).

Pichot J., Gilot B., Bancillon B. et Pauget L. Épidémiologie de la maladie de Lyme dans la région Rhône-Alpes (France). Bulletin de la Société Française de Parasitologie, 1990, 8, supplément 2, 1186.

Rey-Coquals A. Culture et identification de Borrelia burgdorferi chez Ixodes ricinus (Acaria Ixodidae). Thèse de Doctorat en Pharmacie, Lyon 1991.

Rioux J.A., Lanotte G., Cousserans J. el Gabinaud J.J. Écologie, développement et santé publique. Foyers d'infection et parasitocènoses. Stratégies d'enquête et d'intervention. Colloque "Écologie et Développement". Éditions du CNRS, 1979, 19-20 sept., 241-284.

Roman E., Lu-Hurnh-Thanh et Pichot J. Étude biogéographique et écologique sur les tiques (Acariens, Ixodidae) de la région lyonnaise. Bulletin Mensuel de la Société Linné Lyonnaise, 1973, nºpécial, 6-73.

Tremel. N. Contribution à l'étude de la Borréliose de Lyme dans les massifs forestiers de la Région Parisienne. D. E. A. Interaction Hôtes-Parasites, Université Paris XII, 1993, $76 \mathrm{p}$.

Vullermet I. Maladie de Lyme et Ixodes ricinus dans les Alpes du Nord. Thèse de Doctorat en Pharmacie, Lyon 1991. 